DOI:

$10.1038 /$ nrc2093

\section{URLs}

TRF2

http://www.ncbi.nlm.nih.gov/ entrez/query.fcgi?db=gene\&c $\mathrm{md}=$ Retrieve\&dopt=full_ report\&list_uids=7014

$\Rightarrow$

\title{
Going to any length
}

The telomere-binding protein TRF2 (telomeric repeat binding factor 2) is well-known for maintaining the integrity of telomeres, but despite showing increased expression in several human tumours its role in cancer is unclear. Now Maria Blasco and colleagues show that the overexpression of TRF2 is oncogenic in a mouse model of skin carcinogenesis, and telomerase deficiency dramatically potentiates this tumorigenic effect.

The authors had previously shown that mice in which TRF2 was overexpressed in the skin, through the keratin 5 (K5) promoter, were more susceptible to UV-induced skin cancer. To investigate whether TRF2 overexpression is oncogenic in response to different tumorigenic stimuli, the authors investigated a chemically-induced model of multistage carcinogenesis in the skin. They found that K5-Trf2 mice developed more tumours that were often larger in size, and these mice had a higher mortality rate. These findings are surprising, as these mice have short telomeres, which is thought to be a potent tumour-suppressing feature. To clarify how overexpressed TRF2 might affect tumorigenesis the authors crossed K5-Trf2 mice with telomerase-deficient $\left(\mathrm{TerC}^{-/-}\right)$mice, and showed that telomerase loss and TRF2 overexpression resulted in a dramatic increase in both spontaneous and UV-induced tumours.
Cytogenetic analysis of keratinocytes from $\mathrm{TerC}^{-1-}, \mathrm{K} 5-\mathrm{Trf} 2$ and K5-Trf2; $\operatorname{TerC}^{-1-}$ mice showed that TRF2 not only induced telomere shortening and end-to-end chromosomal fusions, which are further increased by telomerase loss, but also extrachromosomal telomeres, interstitial telomeres and multiple telomeres at chromosomal ends that develop independent of telomere length or telomerase activity. This finding indicates that TRF2 overexpression promotes tumorigenesis by inducing chromosomal instability, and suggests that in these conditions - short telomeres and an absence of telomerase - alternative telomere maintenance (ALT) mechanisms are activated. Consistent with this idea, Blasco and colleagues found several features that are characteristic of ALT in K5-Trf2 and K5-Trf2; $\mathrm{Terc}^{-/}$cells.

Given that TRF2 is overexpressed in various human tumours, these results raise an important issue - therapeutic strategies based on telomerase inhibitors might not be effective in TRF2-overexpressing tumours.

\section{Francesca Pentimalli}

ORIGINAL RESEARCH PAPER Blanco, R., Munoz, P., Flores, J. M., Klatt, P. \& Blasco M. A. Telomerase abrogation dramatically accelerates TRF2-induced epithelial carcinogenesis. Genes Dev. 21, 206-220 (2007)

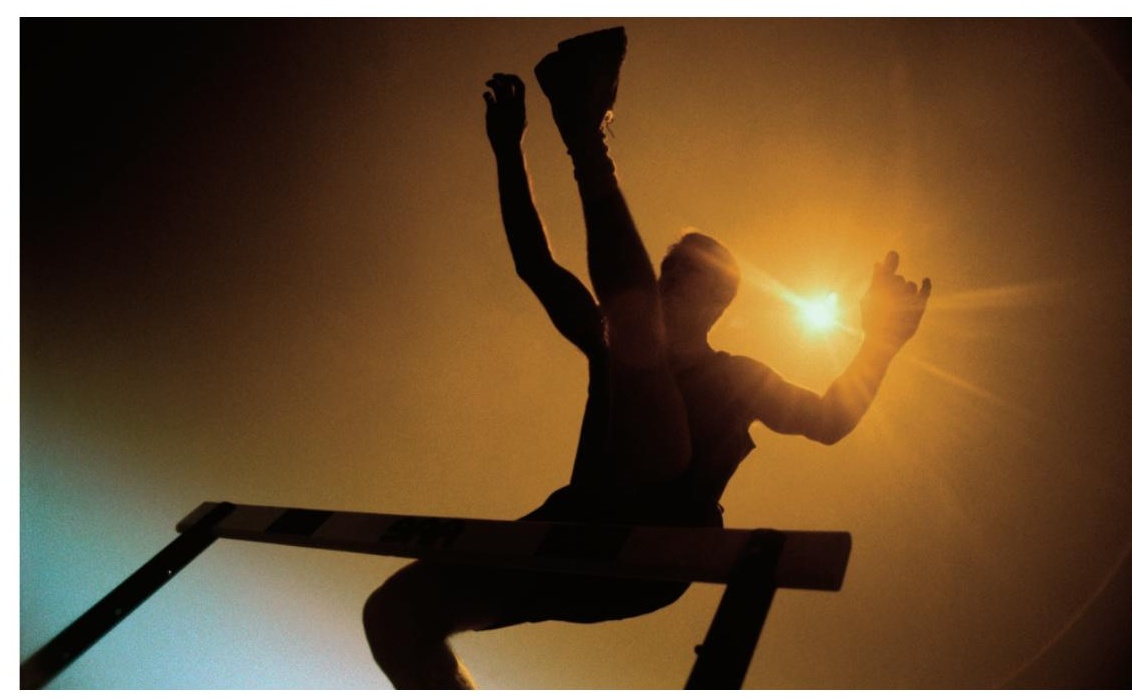

